\title{
High energy particle transport in stochastic magnetic fields in the solar corona
}

\author{
M. Gkioulidou ${ }^{1}$, G. Zimbardo ${ }^{2}$, P. Pommois ${ }^{2, \star}$, P. Veltri $^{2}$, and L. Vlahos ${ }^{1}$ \\ 1 Department of Physics, Aristotle University of Thessaloniki, 54124 Thessaloniki, Greece \\ e-mail: mgioul@physics . auth.gr \\ 2 Dipartimento di Fisica, Università della Calabria, Ponte P. Bucci, cubo 31C, 87036 Arcavacata di Rende, Italy \\ e-mail: zimbardo@fis.unical.it
}

Received 1 June 2006 / Accepted 12 September 2006

\begin{abstract}
Aims. We study energetic particle transport in the solar corona in the presence of magnetic fluctuations by analyzing the motion of protons injected at the center of a model coronal loop.

Methods. We set up a numerical realization of magnetic turbulence, in which the magnetic fluctuations are represented by a Fourier expansion with random phases. We perform test particle simulations by varying the turbulence correlation length $\lambda$, the turbulence level, and the proton energy. Coulomb collisions are neglected.

Results. For large $\lambda$, the ratio $\rho / \lambda$ (with $\rho$ the Larmor radius) is small, and the magnetic moment is conserved. In this case, a fraction of the injected protons, which grow with the fluctuation level, are trapped at the top of the magnetic loop, near the injection region, by magnetic mirroring due to the magnetic fluctuations. The rest of the protons propagate freely along $\boldsymbol{B}$, corresponding to nearly ballistic transport. Decreasing $\lambda$, that is, increasing the ratio $\rho / \lambda$, the magnetic moment is no longer well conserved, and pitch angle diffusion progressively sets in. Pitch angle diffusion leads to a decrease in the trapped population and progressively changes proton transport from ballistic to superdiffusive, and finally, for small $\lambda$, to diffusive.

Conclusions. Particle mirroring by magnetic turbulence makes for compact trapping regions. The particle dynamics inside the magnetic loop is non-Gaussian and the statistical description of transport properties requires the use of such ideas as the Lévy random walk.
\end{abstract}

Key words. Sun: corona - turbulence - diffusion - Sun: flares

\section{Introduction}

It is widely accepted that during a solar flare a large number of electrons, protons, and heavy ions are accelerated around the top of the closed magnetic field lines (coronal arcades). Despite the rapid progress made in the last few years on the mechanisms for particle acceleration, the details for particle acceleration during a solar flare remain an open issue (Miller et al. 1997; Cargill 2002). According to the existing observations, the high energy particles accelerated during a solar flare can be split into three distinct classes: (1) electrons and ions with relatively small pitch angle are freely streaming towards the dense and cold parts of the solar atmosphere, where high density thermalizes all particles very rapidly, causing bremsstrahlung that is recorded as hard X-rays and $\gamma$-rays (Vilmer \& MacKinnon 2003); (2) a smaller population of high pitch angle electrons and ions are trapped inside the closed magnetic field lines at the coronal part of the loop radiating gyrosynchrotron emission in radio wave lengths (Melrose \& Brown 1976; Kundu \& Vlahos 1982; Bastian et al. 1998; Fleishman \& Melnikov 1998); (3) a third and very important population may escape from the loop and it is recorded in space and called the Solar Energetic Particles (SEP) (Reames \& Tylka 2002; Cane \& Erickson 2003). Transport and kinematics of all these three populations (trapped, precipitating, and escaping) is an integral part of the theory of particle acceleration.

* Also at Center for High Performance Computing, Università della Calabria, Cosenza, Italy.
Most studies on stochastic particle acceleration assumed relatively simple magnetic topologies (e.g., simple magnetic loops) and the presence of low frequency MHD waves (Miller et al. 1990; Petrosian 1999). The Fokker-Planck equation was used extensively for the analysis of the evolution of the high energy particles inside magnetic traps (e.g., Fletcher 1997). On the other hand, it is well known that irregular photospheric motions create stochastic magnetic field lines above the photosphere, so that the transport properties of the particles will be radically different. Cross-field transport of thermal electrons in a coronal loop in the presence of tangled magnetic fields was recently considered by Galloway et al. (2006).

The influence of stochastic magnetic fields on particle transport is the subject of this study. We explore the propagation of energetic protons in the presence of magnetic turbulence inside an open coronal loop. We set up a numerical realization of magnetic fluctuations and inject a number of protons to study their evolution over time. Energetic protons are considered as test particles. Of course, the test particle approach is only an approximation, as it is clear that turbulent transport will influence the global plasma structure and even the turbulence properties. However, the self-consistent solution of the turbulent transport problem constitutes a formidable theoretical and numerical challenge. In the case of energetic particles in coronal loops, e.g., $1 \mathrm{MeV}$ protons, we can assume that the turbulence properties are related to the thermal plasma component, so that a test particle approach can be appropriate. In the present study, Coulomb collisions are 
neglected. For instance, for $1 \mathrm{MeV}$ protons streaming in an ambient plasma with density $n_{\mathrm{e}}=10^{10} \mathrm{~cm}^{-3}$ and temperature $T \simeq$ $10^{6} \mathrm{~K}$, the collisional mean free path is larger than $400000 \mathrm{~km}$ (e.g., Krall \& Trivelpiece 1972). This is longer than most coronal loops, so that, in the numerical study, collisions are neglected, although some effects of collisions are considered in the discussion. On the other hand, for say, $20 \mathrm{keV}$ electrons, the collisional mean free path is of the order of $60000 \mathrm{~km}$, so that collisions are more relevant to the electron dynamics. Collisional transport of non-thermal electrons in coronal loops has been considered by Fletcher (1995, 1996).

\section{Numerical model}

We perform a test particle simulation in which the ions move in a background field $B_{0} \boldsymbol{e}_{z}$ plus a fluctuating field $\delta \boldsymbol{B}(\boldsymbol{r})$. We use a numerical realization of a fully three dimensional turbulent magnetic field, to study the evolution of magnetic field structures. It is basically the same model as Pommois et al. $(1998,1999,2001)$, which proved effective in assessing the influence of turbulence anisotropy on transport properties, and which we recall here for completeness. The magnetic fluctuations are constructed in a parallelepipedal box with sides $\ell \sim 3 \lambda$, where $\lambda$ is the turbulence correlation length, and are given by $\delta \boldsymbol{B}(\boldsymbol{r})=\sum_{\boldsymbol{k}, \sigma} \delta B(\boldsymbol{k}) \mathbf{e}^{(\sigma)}(\boldsymbol{k}) \operatorname{expi}\left[\boldsymbol{k} \cdot \boldsymbol{r}+\phi_{\mathbf{k}}^{(\sigma)}\right]$, where $\boldsymbol{e}^{(\sigma)}(\boldsymbol{k})$ are the polarization vectors and $\phi_{\mathbf{k}}^{(\sigma)}$ are random phases. The Fourier coefficients are given by

$\delta B(\boldsymbol{k})=\frac{C}{\left(k_{x}{ }^{2} \lambda^{2}+k_{y}{ }^{2} \lambda^{2}+k_{z}{ }^{2} \lambda^{2}\right)^{\alpha / 4+1 / 2}}$,

where $C$ is a normalization constant and the adopted spectral in$\operatorname{dex}$ is $\alpha=3 / 2$. With this value of $\alpha$, the correlation length $\lambda$ agrees with the longest wavelengths $\lambda_{\max }$ present in the turbulence. Note that with this choice of $\delta B(\boldsymbol{k})$, corresponding to the case of isotropic turbulence, the Fourier amplitude isolevels are spherical surfaces in $\boldsymbol{k}$ space. The wave vectors are chosen on a grid as $\boldsymbol{k}=2 \pi\left(n_{x} / \lambda, n_{y} / \lambda, n_{z} / \lambda\right) / N_{\min }$ and the spectrum has a cut-off for both the short and the long wavelengths (band spectrum) with $N_{\min }^{2} \leq n_{x}^{2}+n_{y}^{2}+n_{z}^{2} \leq N_{\max }^{2}$. For the present runs, $N_{\text {min }}=3$ and $N_{\text {max }}=16$. In this paper, we consider isotropic turbulence, with wave vectors distributed in three dimensions (3D). Anisotropic turbulence can also be described by our numerical model, but we leave this for a future study. A sketch of magnetic field lines in the presence of turbulence is shown in Fig. 1.

To trace particle trajectories in the above turbulent magnetic field, we have to numerically solve the equations of motion:

$\frac{\mathrm{d} \boldsymbol{r}}{\mathrm{d} t}=\boldsymbol{v}$

$\frac{\mathrm{d} \boldsymbol{v}}{\mathrm{d} t}=\frac{q}{m c \gamma_{\mathrm{rel}}} \boldsymbol{v} \times \boldsymbol{B}$

where $\boldsymbol{v}$ is the particle velocity, $\gamma_{\mathrm{rel}}$ is the Lorentz factor, and $\boldsymbol{B}$ is the total magnetic field. We consider energetic particles with velocity $v$ much larger than the Alfvén velocity. Typically, for $1 \mathrm{MeV}$ protons the particles move at $v \simeq 14000 \mathrm{~km} \mathrm{~s}^{-1}$, which is much larger than the typical Alfvén speed in the corona with mean magnetic field $\sim 10 \mathrm{G}$ and density $n_{\mathrm{e}} \sim 10^{10} \mathrm{~cm}^{-3}$. When the particle's velocity is much larger than the Alfvén speed, the time evolution of magnetic fluctuations can be neglected, so that the above magnetostatic approximation is justified. In the same limit, the electric force due to the electric

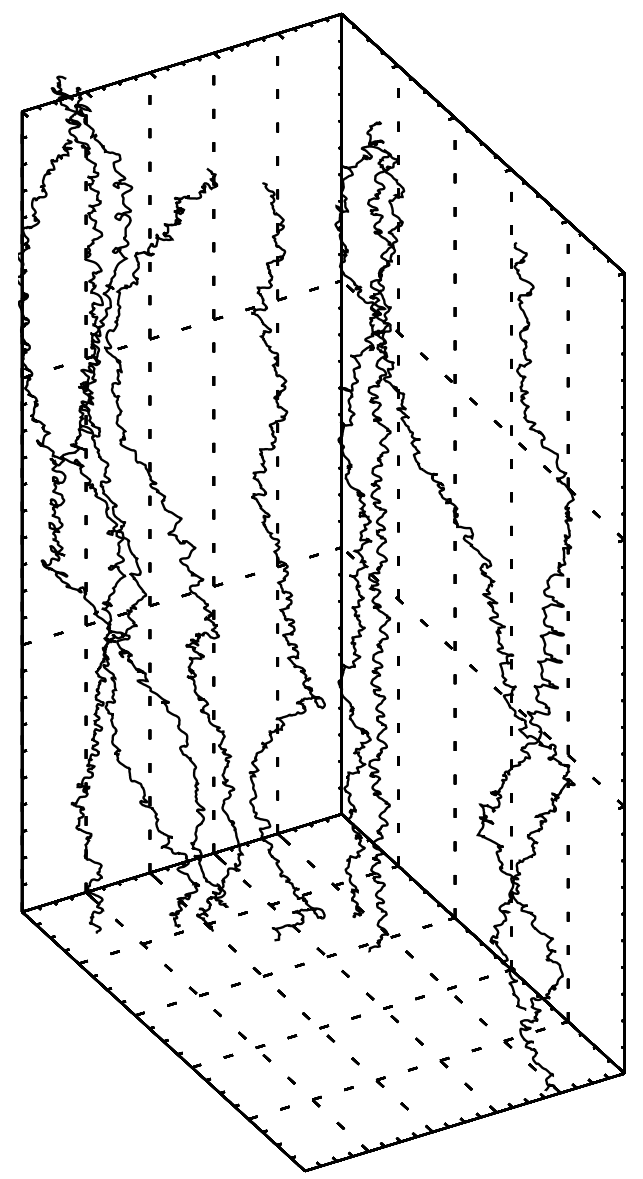

Fig. 1. Plot of sample field lines inside the loop simulation box, for $\delta B / B_{0}=0.2$ (axes not to scale).

field $\delta E$ of the MHD waves, which is of order of $\delta E \simeq V_{\mathrm{A}} \delta B / c$, can be neglected.

We set up a rectangular simulation box that models an idealized coronal loop. We take the loop length $L_{z}=30000 \mathrm{~km}$, and the loop thickness $L_{x}=1000 \mathrm{~km}$. In the simulation, all lengths are normalized to the loop thickness $L_{x}=L$, so that the simulation box extends from 0 to $1 L$ in $x$ and $y$, and from $-15 L$ to $15 L$ in $z$. Further, velocities are normalized to $v_{\mathrm{n}}=14000 \mathrm{~km} \mathrm{~s}^{-1}$, and times to the inverse proton gyrofrequency, $\Omega_{i}{ }^{-1}$. We can assume a typical value of $B_{0}=10$ Gauss, so that $\Omega_{i}^{-1} \simeq 10^{-5} \mathrm{~s}$. The numerical integration is performed with a Runge-KuttaFehlberg algorithm of the fourth-fifth order with adaptive steps. To obtain good numerical accuracy, an integration step of at most $10^{-1} \times \Omega_{i}^{-1}$ was chosen. This means that over 60 integration steps are necessary to complete a gyroorbit. Since there is no electric field, the accuracy of the integration can be checked by means of the conservation of kinetic energy. Several checks were made for different particle energies, adjusting the accuracy parameter of the integration algorithm to ensure that the relative error on energy be less than $10^{-6}$, and typically much less.

Ideally, the magnetic turbulence wavelengths in the loop can extend from the loop thickness $\sim L$ down to the dissipation scale in the corona, which can be estimated as $\lambda_{\text {diss }}=\rho_{\text {th }}$, where $\rho_{\text {th }}$ is the Larmor radius of thermal protons. Because of the finite numerical resources, we are not able to simulate such a long spectrum with our fully $3 \mathrm{D}$ realization of the turbulence. On the other hand, the particle interaction with inhomogeneous fields sensitively depends on the ratio $\rho / \lambda$, where $\rho$ is the ion Larmor radius (e.g., Zimbardo et al. 2006). To explore the influence of the 
Table 1. Run parameters.

\begin{tabular}{ccccc}
\hline \hline Run & $\ell / L$ & $\delta B / B_{0}$ & $\rho / \lambda_{\max }$ & $\rho / \lambda_{\min }$ \\
\hline 1a & 1 & 0.05 & $4.2 \times 10^{-4}$ & $2.24 \times 10^{-3}$ \\
1b & 1 & 0.1 & $4.2 \times 10^{-4}$ & $2.24 \times 10^{-3}$ \\
1c & 1 & 0.2 & $4.2 \times 10^{-4}$ & $2.24 \times 10^{-3}$ \\
2a & 0.1 & 0.1 & $4.2 \times 10^{-3}$ & $2.24 \times 10^{-2}$ \\
2b & 0.1 & 0.1 & $1.4 \times 10^{-2}$ & $7.3 \times 10^{-2}$ \\
2c & 0.1 & 0.1 & $4.2 \times 10^{-2}$ & $2.24 \times 10^{-1}$ \\
3a & 0.01 & 0.05 & $4.2 \times 10^{-2}$ & $2.24 \times 10^{-1}$ \\
3b & 0.01 & 0.1 & $4.2 \times 10^{-2}$ & $2.24 \times 10^{-1}$ \\
3c & 0.01 & 0.2 & $4.2 \times 10^{-2}$ & $2.24 \times 10^{-1}$ \\
4 & 0.001 & 0.1 & $4.2 \times 10^{-1}$ & 2.24 \\
\hline
\end{tabular}

different wavelengths on proton transport, we change the ratio between the Larmor radius and the turbulence maximum (minimum) wavelength $\lambda_{\max }\left(\lambda_{\min }\right), \rho / \lambda_{\max }$, and $\rho / \lambda_{\min }$, in the different runs as indicated in Table 1 . In the simulation, this change of correlation length is obtained by changing the ratio between the side $\ell=3 \lambda_{\max }$ of the magnetic fluctuation box and the side $L$ of the loop simulation box. Runs with different values of the ratio $Q=\ell / L$ and different proton energies have been done.

\section{Particle dynamics}

We inject protons at the center of the simulation box, at $z=0$ and $x, y$ in a circle of radius $r=0.05 L$, with velocities in random directions and corresponding to an energy shell of $1 \mathrm{MeV}$ (except for the cases indicated below). For reference, we consider proton dynamics; however, the behavior of the other ion species can be understood by making reference to the ratio $\rho / \lambda_{\max }$, reported in Table 1, since no electric force and no time dependence of $\delta B$ are included in our simulation. We have performed several runs changing the turbulence level, the turbulence correlation scale $\ell / L$, and the proton energy $E$. In particular, $E=10 \mathrm{MeV}$ for run $2 \mathrm{~b}$, and $E=100 \mathrm{MeV}$ for run $2 \mathrm{c}$. The typical number of injected protons is approximately 1000 . For these runs the total integration time was set to $t_{\max }=2 \times 10^{6} \Omega_{i}^{-1}$. For the solar corona, we assume typical values of $\delta B / B_{0}=0.1-0.2$, although different values have also been used, as indicated in Table 1 .

\subsection{Cross magnetic field transport}

In Fig. 2 we present the projection of protons at $z= \pm 15 L$ on the $x y$ plane for $\ell / L=1$, to show the cross magnetic field transport of the protons in relation with the fluctuation level. For these specific runs we used a larger injection radius, $r=0.1 L$, to have an injection region of size comparable to the turbulence correlation length $\lambda$. It can be seen that the number of protons exiting from the edges, that is from $x, y=0-1 L$, increases with the fluctuation level. If our magnetic field topology were different this number might have been even larger, since it depends on the number of the open magnetic field lines that is included in the simulation box. The plots of Fig. 2 are in agreement with the fact that the transport perpendicular to the average magnetic field $\boldsymbol{B}_{0}$ grows with $\delta B / B_{0}$; if we assume that the magnetic field line transport corresponds to the quasilinear regime, we can conclude that the magnetic field line diffusion coefficient scales as $D_{\mathrm{m}} \sim\left(\delta B / B_{0}\right)^{2} \lambda$ (Zimbardo et al. 1995, 2000; Pommois et al. 2001; Galloway et al. 2006). The latter expression also shows that perpendicular diffusion is proportional to the turbulence correlation length. For $\delta B / B_{0} \simeq 0.1$ and isotropic turbulence, the magnetic configuration corresponds to only partial stochasticity
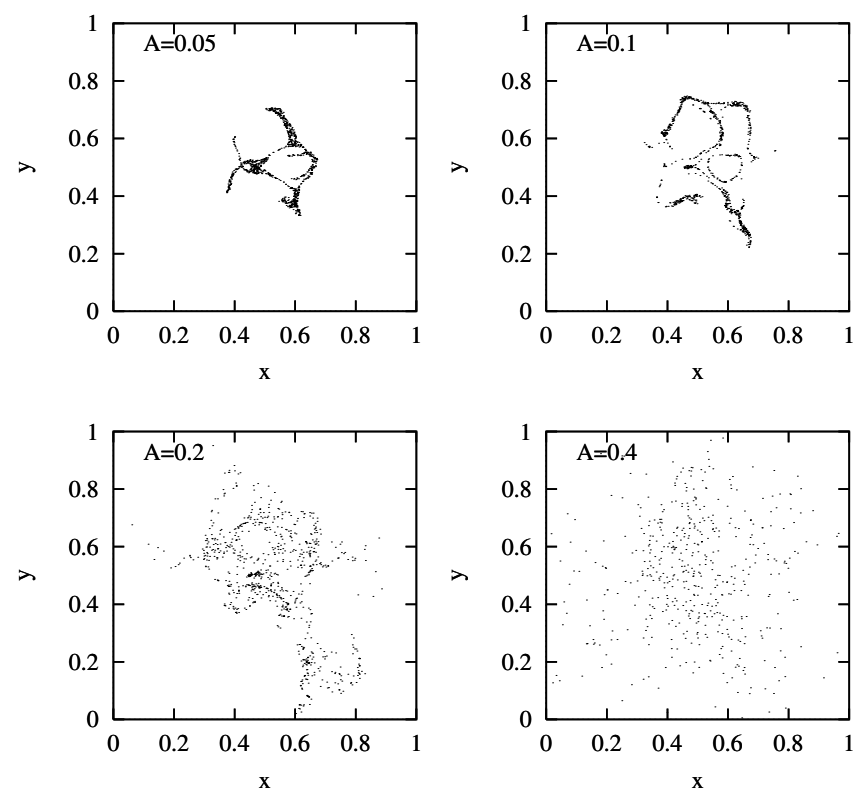

Fig. 2. Projection of protons' positions on the $x y$ plane for $\ell / L=1$ and $A \equiv \delta B / B_{0}=0.05,0.1,0.2,0.4$.

(Zimbardo et al. 1995, 2000). In such a case, particles and field lines move fast in the stochastic layer in between closed magnetic surfaces (see Fig. 2). This motion is not truly random, but chaotic, and can give rise to directed transport (Zimbardo et al. 2004; Zimbardo 2005). The non uniform, "intermittent" distribution of protons in Fig. 2 is an example of non-Gaussian transport. It is interesting to compare Fig. 2 with standard, Gaussian diffusion: in such a case, after a "collision", particles can move in arbitrary directions, thus smoothing out the density gradients. Such a random motion corresponds to a density distribution of, say, energetic particles injected at $\boldsymbol{r}_{0}$ described by a Gaussian distribution like

$n(\boldsymbol{r}, t)=n_{0}(2 \pi \kappa t)^{-3 / 2} \exp \left[-\left(\boldsymbol{r}-\boldsymbol{r}_{0}\right)^{2} /(2 \kappa t)\right]$

where $\kappa$ is the particle diffusion coefficient. Conversely, in the case of turbulent diffusion, motion can exhibit memory effects (which in the present case are due to the magnetic structure), transport is fast in the stochastic layer, but not isotropic, so that density gradients are not necessarily smoothed out (Mazur et al. 2000; Ruffolo et al. 2003; Zimbardo et al. 2004), as shown in Fig. 2. In such a case, the density distribution is not Gaussian, and its description may require the use of Lévy distribution functions and fractal geometry (Bouchaud \& Georges 1990; Zimbardo 2005).

\subsection{Parallel propagation and trapped particles}

We found that, especially for large values of $\ell / L$, a large fraction of protons are trapped by the magnetic fluctuations, which locally create magnetic mirrors. Sample trajectories of trapped and non-trapped protons are shown in Fig. 3. We can argue that the dynamics of the trapped protons is radically different from that of non-trapped protons. The former remain localized for long times near the injection point (although protons can slowly leak out of the mirror regions because of the $\nabla B$ drift and the stochasticity of field lines, as well as because of collisions), while the latter propagate relatively freely along the magnetic field, with some modulation of $v_{\|}$because of the variations of $|\boldsymbol{B}|$, so that these particles reach the loop footpoints in a relatively short time. 

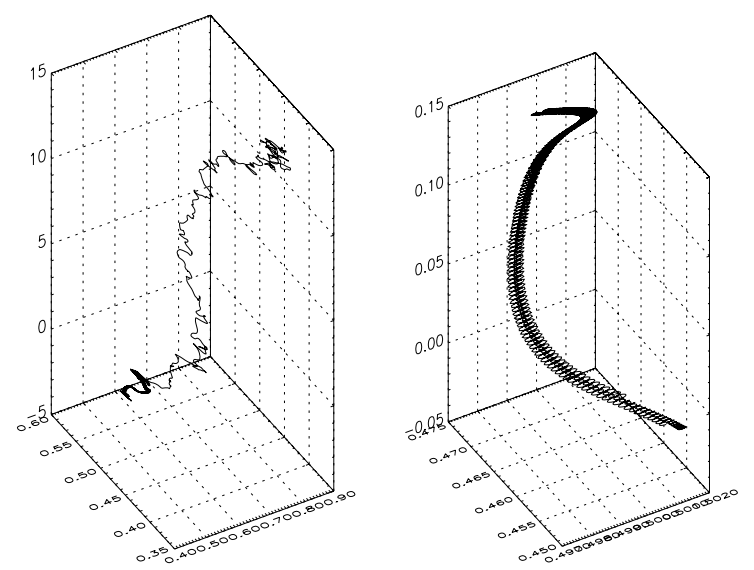

Fig. 3. Proton trajectories obtained for $\delta B / B_{0}=0.2$ and $\ell / L=1$. On the left, trajectory of a proton that is not trapped. On the right, trajectory of a trapped proton. Note the very different axis labels, and, on the right, the multiple reflections at the mirror points and the small lateral displacement of the guiding center due to $\nabla B$ drift. Axes not to scale.
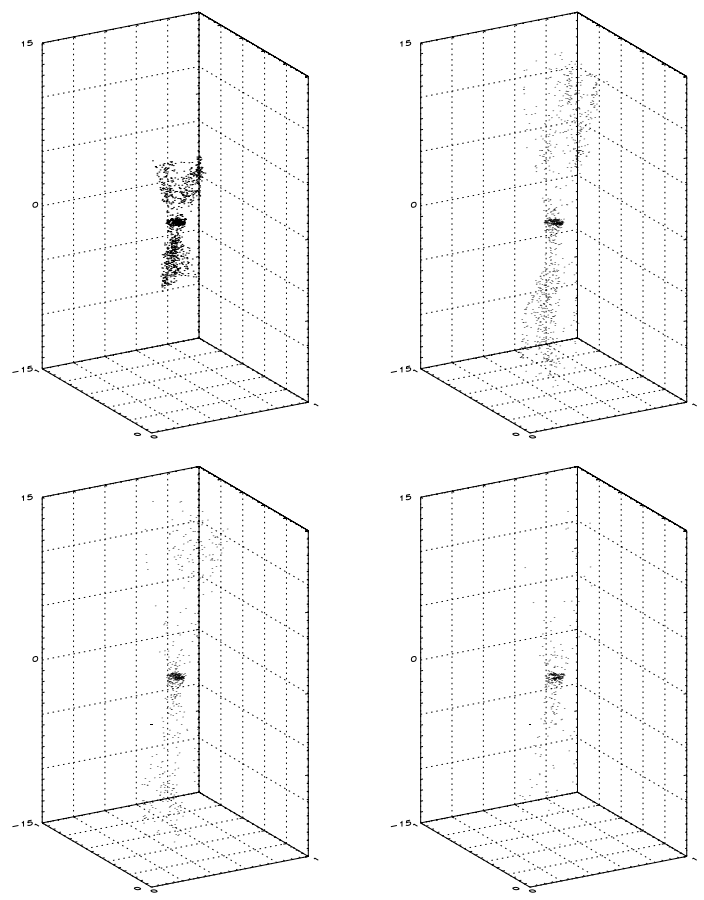

Fig. 4. "Snapshots" of proton positions at times $t_{1}=6 \times 10^{4} \Omega_{i}^{-1}, t_{2}=$ $12 \times 10^{4} \Omega_{i}^{-1}, t_{3}=20 \times 10^{4} \Omega_{i}^{-1}$, and $t_{4}=60 \times 10^{4} \Omega_{i}^{-1}$, for $\ell / L=1$. Axes not to scale.

We can see that protons are trapped in a region whose size, parallel to the background field $B_{0}$, is of the order of the turbulence correlation length $\lambda$, which also is the scale of the magnetic mirror due to magnetic fluctuations. For instance, the trajectory in the left panel of Fig. 3 has a vertical size $\Delta z \simeq 0.2 L$, comparable to $\lambda=L / 3$. This implies that in the trap-plus-precipitation model, the size of the trapping region may be set by the turbulence correlation length. The different types of proton dynamics can be visualized by plotting the proton positions in the simulation box at various fixed times after injection: in Fig. 4 we show four such "snapshots" for $\delta B / B_{0}=0.1, \ell / L=1$, and $E=1 \mathrm{MeV}$. After some time, we can see that the trapped particles and the free-streaming particles form two different populations. On the other hand, Fig. 5 shows the corresponding "snapshots" (although at later times because of the much slower
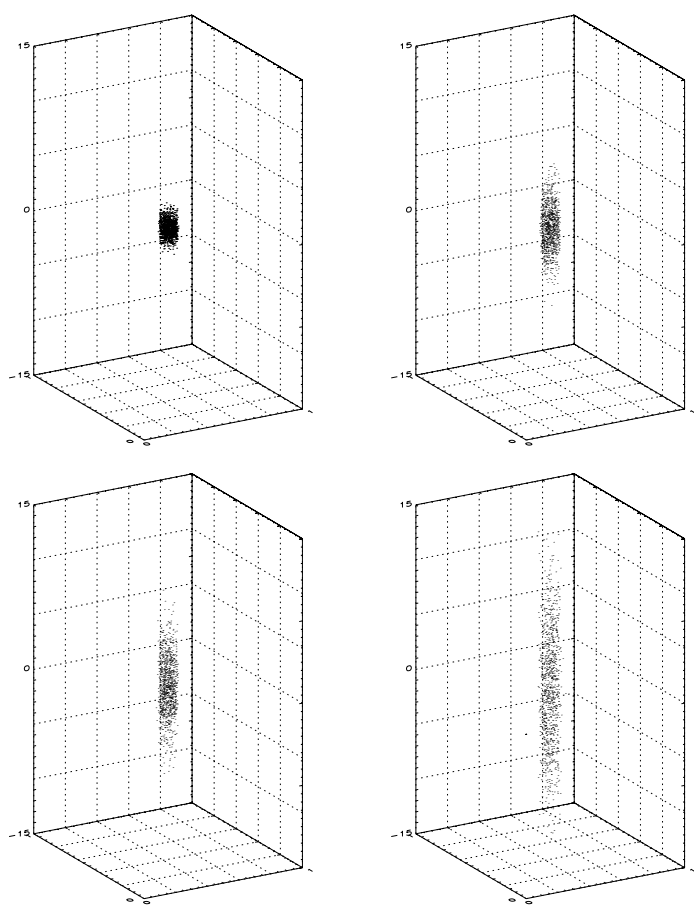

Fig. 5. "Snapshots" of proton positions at times $t_{1}=6 \times 10^{4} \Omega_{i}^{-1}, t_{2}=$ $30 \times 10^{4} \Omega_{i}^{-1}, t_{3}=50 \times 10^{4} \Omega_{i}^{-1}$, and $t_{4}=200 \times 10^{4} \Omega_{i}^{-1}$, for $\delta B / B_{0}=0.1$ and $\ell / L=0.001$.

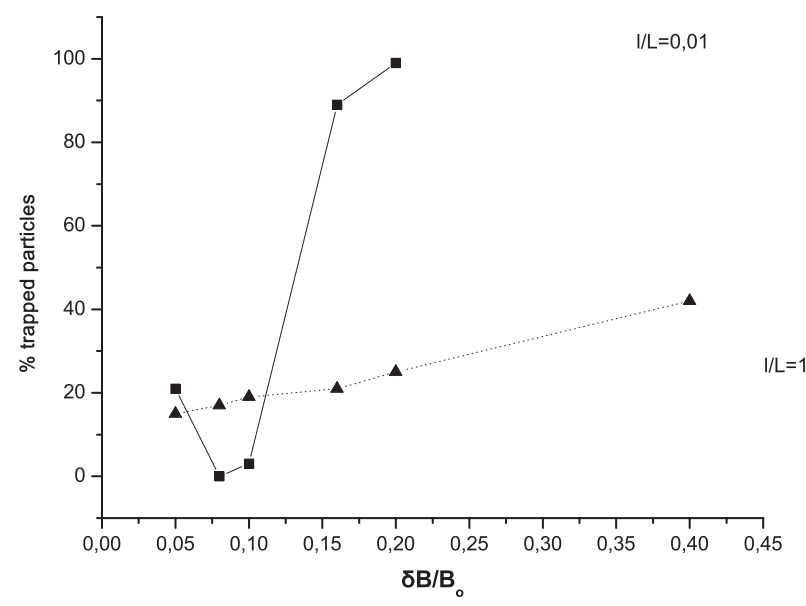

Fig. 6. Percentage of trapped protons versus the fluctuation level.

propagation) for $\ell / L=0.001$. In such a case, motion is globally diffusive, trapping is unimportant, and proton positions correspond to a nearly Gaussian distribution.

In Fig. 6 we plot the dependence of the percentage of trapped protons as a function of $\delta B / B_{0}$ for two different cases, $\ell / L=1$ and $\ell / L=0.01$. For $\ell / L=1$, as the fluctuation level increases, the percentage of trapped protons increases as well, since magnetic mirroring becomes more intense. On the other hand, for $\ell / L=0.01$, the percentage of trapped protons decreases as the fluctuation level increases from 0.05 to 0.1 . In these cases, $\rho / \lambda_{\min }=2.24 \times 10^{-1}$ (see Table 1$)$, so that the magnetic moment is no longer conserved: efficient pitch angle scattering is found, and this scattering is faster when $\delta B / B_{0}$ is increased. As a consequence of increasing $\delta B / B_{0}$, a smaller fraction of protons is trapped by magnetic mirroring, due to stronger pitch angle diffusion. However, increasing the fluctuation level further, pitch angle diffusion is so fast that parallel motion becomes diffusive (see 


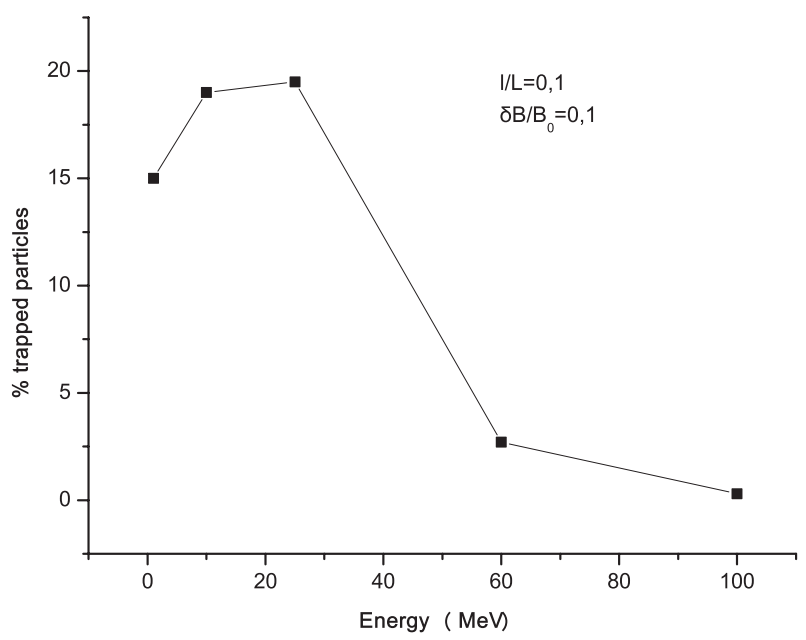

Fig. 7. Percentage of trapped protons versus the proton energy.
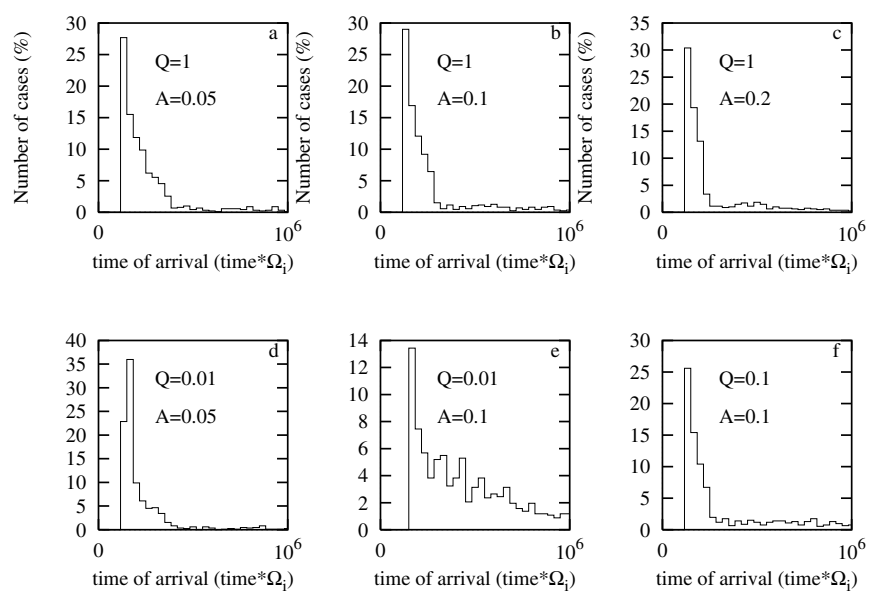

Fig. 8. Histograms representing the arrival time profile of $1 \mathrm{MeV}$ protons at $z= \pm 15$. The parameters of each run are indicated in each panel.

and $Q=\ell / L$, and they mimic the time profile of the emission from the loop foot points, assuming that the emission is histograms were done for protons exiting from $z=+15 L$ and from $z=-15 L$ : these exhibit the same overall behavior, which can be taken as an indication that the number of injected protons is statistically enough. Here we present only the histograms for the total number of protons exiting along $z$. In the panels of Fig. 8 and following, we have indicated, for brevity, the ratio of spatial scales as $Q=\ell / L$, and the fluctuation amplitude as $A=\delta B / B_{0}$.

We can see that for large correlation lengths, most protons arrive promptly (scatter-free) to the foot points, while trapped protons are not exiting at all. The upper row of Fig. 8, panels a, $\mathrm{b}$, and $\mathrm{c}$, shows the time profile for $\ell / L=1$ and for different fluctuation levels (as indicated): we can see that when increasing the fluctuation level, fewer protons are arriving at middle times because more protons are trapped. In particular, the trapped protons have a large pitch angle, that is, a small $v_{\|}$. Increasing $\delta B / B_{0}$, reduction in the number of protons in the tail of the time profile. A different phenomenology is found increasing $\delta B / B_{0}$ for shorter correlation lengths: panels $\mathrm{d}$ and e of Fig. 8 show the time profiles for $\ell / L=0.01$ and $\delta B / B_{0}=0.05,0.1$, respectively. It can be seen that increasing the fluctuation level, a longer tail is created in the time profile: in these cases pitch angle diffusion increases with the fluctuation level, and proton motion along $z$ becomes diffusive (that is slower than scatter-free). Finally, panels $\mathrm{b}, \mathrm{f}$, and e give the time profile for $\ell / L$ varying from 1 to 0.1 and to 0.01 , respectively, for constant fluctuation level $A=0.1$ (we have found it impractical to obtain the time profiles for $\ell / L=0.001$, as the very slow diffusive motion would require even longer integration times to have enough arriving protons). We can see clearly that decreasing the correlation length, a tail of "late" protons develops: this "tail" is the result of pitch angle diffusion, which slows down the propagation along $z$.

In summary, the effect of increasing $\delta B / B_{0}$ is twofold: it causes a decrease of the tail of the time profile for large turbulence correlation lengths (because of enhanced trapping of particles with small $v_{\|}$), and it causes the increase of the tail of the time profile for small turbulence correlation lengths (because of enhanced pitch angle diffusion).

We can argue that the upper row of Fig. 8 qualitatively describes the behavior of energetic electrons, too. For instance, for $20 \mathrm{keV}$ electrons, $B_{0}=10 \mathrm{G}$, and $\ell / L=1$, directly proportional to the number of arriving protons. Separate protons with somewhat larger $v_{\|}$are trapped, and this causes a at $z= \pm 15 L$ versus time. These histograms are shown in Fig. 8 for $1 \mathrm{MeV}$ protons and for different values of $\delta B / B_{0}$ depends not only on $\rho / \lambda_{\min }$, but also on the turbulence level, as shown in Fig. 6 and, below, in Fig. 10.

Further insight on proton parallel motion can be obtained by making the histograms of the number of protons arriving later), which is slower than free-streaming. In such a case, a large
number of protons are reported as trapped since these protons remain inside the simulation box, although they are not trapped by
magnetic mirroring. In other words, these protons propagate in a diffusive way, but do not have enough time, in the simulation, of the protons are trapped (not shown). However, they are not trapped by magnetic mirrors, but simply, pitch angle scattering is very efficient and the parallel mean free path is very short, so case, trapping is found when $\rho / \lambda_{\min } \ll 1$, so that the smallest wavelength (rather than the loop radius) gives the scale that quasi-neutral sheet of thickness $L_{z}$, Buechner \& Zelenyi (1989) have shown that motion is adiabatic when $\rho / R_{\mathrm{c}} \ll 1$, with nent of the center of the current sheet (here, $B_{\mathrm{n}}$ is the compothe asymptotic field out of the current sheet). When $\rho / R_{\mathrm{c}}$ particles are trapped by bouncing motion in the magnetic mirror due to $B_{0}$ and $B_{\mathrm{n}}$. When $\rho / R_{\mathrm{c}} \sim 1$, the adiabatic invariants are no longer conserved and particles undergo chaotic motion and , 
we obtain $\rho_{\mathrm{e}} / \lambda \simeq 1.5 \times 10^{-6}$, with $\rho_{\mathrm{e}}$ the electron gyroradius. Comparing this estimate with the values of $\rho / \lambda$ in Table 1 , we can see that electron motion is adiabatic in most cases, so that electrons are subject to magnetic mirroring, but not to pitch angle diffusion (save for collisions).

\section{Statistical properties}

In the previous section we analyzed proton transport by means of several diagnostics: these have evidenced that qualitatively different dynamics can be obtained, i.e., scatter-free propagation, trapped motion, and diffusive motion, depending on the values of $\ell / L, \delta B / B_{0}$, and proton energy. Here we want to characterize these different behaviors by means of their statistical properties in the time asymptotic regime (that is for long enough times).

\subsection{Theoretical analysis}

Scatter-free or, in the opposite case, trapped dynamics correspond to nondiffusive, non-Gaussian behaviors, which can be described by a generalized transport law

$\left\langle\left(\Delta r_{i}\right)^{2}\right\rangle=2 \kappa_{i} t^{\gamma_{i}}$,

where $i=x, y, z$ and $\Delta r_{i}=r_{i}-r_{i 0}$, with $r_{i 0}$ the initial proton position. Here, $\kappa_{i}$ is a generalized transport coefficient, corresponding to the standard diffusion coefficient when $\gamma_{i}=1$, and $\gamma_{i}$ is the anomalous transport exponent. We note that $\gamma_{i}=2$ corresponds to ballistic transport (i.e., purely scatter-free), $1<\gamma_{i}<2$ corresponds to superdiffusion, which can be described statistically by means of a Lévy random walk (Klafter et al. 1987; Bouchaud \& Georges 1990; Klafter et al. 1996; Metzler \& Klafter 2000), while $\gamma_{i}<1$ corresponds to subdiffusion and trapping. A Lévy random walk is a transport process in which the free path lengths $x$ have a power law probability distribution $\psi(x, t)$ for large $x$, so that very long free paths have a small but non negligible probability (e.g., Bouchaud \& Georges 1990). This is at variance with what is found in the case of Gaussian statistics, where $\psi(x, t) \propto \exp \left(-x^{2}\right)$, so that very long displacements are very unlikely. In the case of a power law distribution for free path lengths, $\psi(x) \propto x^{-1+\beta}$ for $x \rightarrow \infty$, the variance of the free path lengths can be diverging. However, a space-time coupling is found in the case of a physical walker, that is, for a material particle that would not cover an arbitrarily long distance in a fixed time, so that longer paths require a longer time. This corresponds to the space-time coupling of Lévy random walks, whereas in the so-called Lévy flights, the length of jumps is independent of the time. In the case of linear coupling between space and time, $\psi(x, t) \propto x^{-(1+\beta)} \delta(t-x / v)$, superdiffusion with $\gamma=3-\beta$ is found for $1<\beta<2$ (Klafter et al. 1987). Several examples of Lévy statistics have been found in a variety of systems (Shlesinger et al. 1995; Zaslavsky 2002; Metzler \& Klafter 2004).

For the considered runs, we have computed the mean square displacement appearing in Eq. (5), as well as the higher order moments needed to compute the flatness $F_{i}$ of the distributions. A running fit of the mean square displacement allows us to compute $\kappa_{i}$ and $\gamma_{i}$. The flatness is defined as

$F_{i}=\left\langle\left(\Delta r_{i}\right)^{4}\right\rangle /\left[\left\langle\left(\Delta r_{i}\right)^{2}\right\rangle\right]^{2}$

and is a measure of the departure of the distribution function of proton positions from a Gaussian distribution, which has flatness equal to 3 . An $F_{i}>3$ implies that the tails of the distribution function are higher than for a Gaussian distribution: this
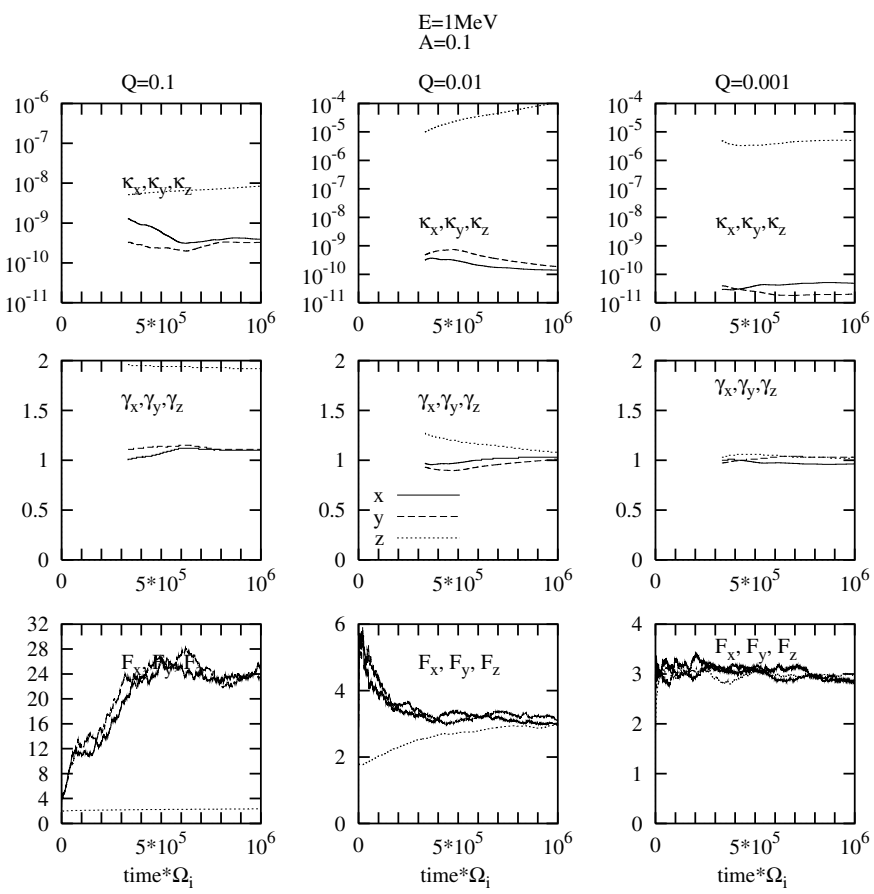

Fig. 9. Running fits of the proton mean square deviations. The top panels give the diffusion constants $\kappa_{i}$, the middle panels the anomalous transport exponents $\gamma_{i}$, and the bottom panels the flatnesses $F_{i}$. The quantities for the different axes are indicated by solid lines $(x)$, dashed lines $(y)$, and dotted lines $(z)$, respectively.

is, for instance, the case of Lévy distribution functions, which have power law tails. On the other hand, $F_{i}<3$ implies that the tails of the distribution are less important than for a Gaussian distribution: this is the case, e.g., of a "rectangular" distribution.

\subsection{Results}

\subsubsection{Statistics for different values of correlation length}

We found that an integration time $t_{\max }=10^{6} \Omega_{i}^{-1}$ is usually sufficient to attain the stabilization of the fitted parameters, after an initial transient. Running fits have been made with a time window of $\Delta t=3 \times 10^{5} \Omega_{i}^{-1}$. The results of the running fits are reported in Fig. 9 for $\ell / L=0.1,0.01,0.001$ and for $\delta B / B_{0}=0.1$. Considering the results in the left panels of Fig. 9, obtained for $\ell / L=0.1$, we can see that transport along the average magnetic field is almost purely ballistic, $\gamma_{z} \simeq 1.92$, while transport perpendicular to $\boldsymbol{B}_{0}$ is weakly superdiffusive, $\gamma_{x} \simeq \gamma_{y} \simeq 1$.1. Ballistic transport corresponds to the fact that protons that are not trapped by magnetic mirroring can freely move along $\boldsymbol{B}$. A similar ballistic transport is found for $\ell / L=1$ (Gkioulidou et al. 2006). Clearly, this kind of transport is very different from standard, Gaussian diffusion. The non-Gaussian nature of transport is confirmed by the values of the flatness, which are very different indeed from the Gaussian values of $3, F_{x} \simeq F_{y} \simeq 25, F_{z} \simeq 2$, (see the bottom left panel of Fig. 9). In particular, $F_{z} \simeq 2$ corresponds to the nearly ballistic parallel motion, so that a large share of protons move like in Fig. 4. Going to smaller turbulence scales, center panels, we find superdiffusive motion along $z, \gamma_{z} \simeq 1.1$, and almost normal diffusion along $x$ and $y$. The superdiffusive motion along $z$ indicates that pitch angle diffusion is at work, but not so much as to imply normal diffusion. The flatnesses are different from 3 in the early phases of transport, but tend to 3 for long 

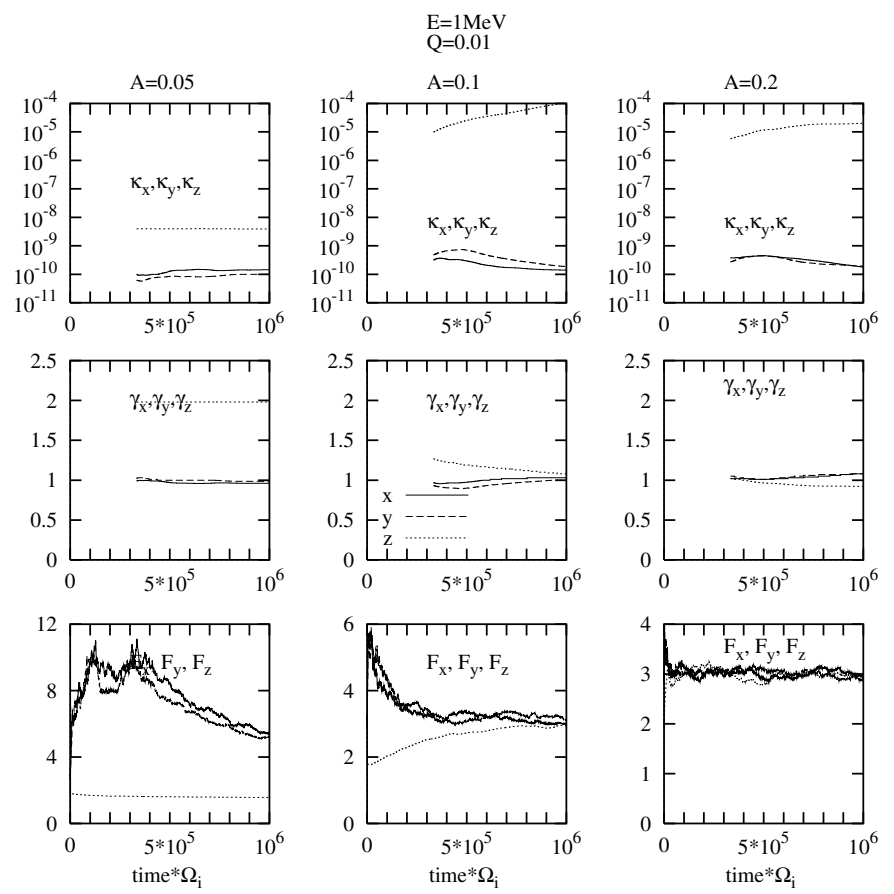

Fig. 10. Same as Fig. 9, but for $\ell / L=0.01$ and $\delta B / B_{0}=0.05,0.1,0.2$. Note the pure ballistic transport along $z$ for $\delta B / B_{0}=0.05$.

times, showing that the departure from the Gaussian regime is not strong. When we decrease the turbulence correlation length further (right panels), we obtain normal, Gaussian diffusion, that is, $\gamma_{x} \simeq \gamma_{y} \simeq \gamma_{z} \simeq 1$ and $F_{x} \simeq F_{y} \simeq F_{z} \simeq 3$. As discussed in Sect. 3 , in this case pitch angle diffusion is very fast, and protons are subject to a random diffusive motion in all directions. Thus, we can see that the character of transport changes with the ratio $\ell / L$, leading to qualitatively different regimes.

\subsubsection{Statistics for different values of turbulence level}

The character of parallel transport dramatically changes with the turbulence level, too: keeping $\ell / L=0.01$, we have done runs with $\delta B / B_{0}=0.05$ and 0.2 . The results, reported in Fig. 10 for $1 \mathrm{MeV}$ protons, show that ballistic transport along $\boldsymbol{B}$ is obtained for $\delta B / B_{0}=0.05, \gamma_{z} \simeq 2$, superdiffusive for $\delta B / B_{0}=0.1$, $\gamma_{z} \simeq 1.1$, and normal diffusion for $\delta B / B_{0}=0.2, \gamma_{z} \simeq 1$. These results show that pitch angle diffusion also depends, of due course, on the turbulence level (that is, on the wave amplitude), so that for weak turbulence levels, scatter-free propagation is obtained, while for larger fluctuation levels normal, Gaussian diffusion is recovered. It is interesting to notice that superdiffusive transport with $\gamma_{z} \simeq 2$ is obtained even in this case when $\rho / \lambda_{\min }=0.224$. Considering that the Larmor orbit diameter is $2 \rho$ and that the peak to valley variation of the wave field corresponds to half a wavelength, we note that $\rho / \lambda_{\min }=0.224$ corresponds to the effective resonance condition. Thus, we can see that superdiffusive transport is obtained even in the case when the particle is in resonance with the turbulence (compare with the results of Zimbardo et al. 2006). On the other hand, perpendicular transport is found to be normal for all the runs from $\delta B / B_{0}=0.05$ to $\delta B / B_{0}=0.2, \gamma_{x} \simeq \gamma_{y} \simeq 1$, although in the former case $F_{x} \simeq F_{y} \simeq 6-8$, while in the latter case $F_{x} \simeq F_{y} \simeq 3$. We also notice that in the globally diffusive cases (right panels of Figs. 9 and 10), the ratio of the diffusion coefficients $\kappa_{x} / \kappa_{z} \simeq 10^{-5}$. We suggest that these relatively small values are due to the small turbulence level.

\section{Discussion and conclusions}

In this paper we have carried out an in-depth investigation of energetic proton transport in the presence of magnetic turbulence, in a configuration corresponding to a model coronal loop. One of the most important points is the formation, because of the considered magnetic fluctuations, of numerous local magnetic traps (see Fig. 4) superimposed on the large-scale magnetic trap expected from the overall magnetic field $B_{0}$ of the loop. The random motions in the photosphere will create large scale stochastic field lines (represented here by the standing waves). These local traps will work on time scales much shorter than the collision time for the ions of $1 \mathrm{MeV}$. Collisions are important for 10-20 keV electrons, but they are not going to dramatically influence the picture presented here. The statistical properties will be the same and their description should include the anomalous transport properties. We have characterized the proton motion by a number of numerical diagnostics, while changing parameters like the turbulence correlation length $\lambda$, the turbulence level $\delta B / B_{0}$, and the proton energy. We can summarize our results as follows:

1. For large $\lambda$, the ratio between the Larmor radius and turbulence wavelength is small, and the magnetic moment is conserved. In this case, a fraction of the protons, which grow with the fluctuation level, are trapped by magnetic mirroring; this population may be responsible for the hard X-ray and, possibly, $\gamma$-ray emission that has been observed close to the loop top (or the injection zone) (Veronig et al. 2005; Barat et al. 1994). The spatial scale for trapping due to mirroring in the magnetic turbulence is of the order of the turbulence correlation length $\lambda$. Another major fraction of protons is not trapped, and propagates freely along $\boldsymbol{B}$. If we neglect collisions, this corresponds to ballistic transport; this population can give rise to electromagnetic emission when reaching the loop footpoints by releasing the particle kinetic energy to the ambient plasma (Vilmer \& MacKinnon 2003).

Previous research has been carried out considering coronal trap models (Alexander 1990; Fletcher 1995, 1996, 1997), without the inclusion of stochastic magnetic fields. In those studies, trapping is due to the large scale magnetic mirror due to the loop structure, and collisions play an important role in causing pitch angle diffusion into the loss cone.

2. Decreasing $\lambda$, that is increasing the ratio $\rho / \lambda$, the magnetic moment is no longer well conserved, and pitch angle diffusion progressively sets in. On the one hand, pitch angle diffusion leads to a decrease of the trapped population; on the other hand, parallel motion is perturbed, and changes progressively from ballistic to superdiffusive, and finally, for small $\lambda$, to diffusive. In the last case, $(\ell / L=0.001)$, parallel motion becomes relatively slow and leads to a very large trapping time of the protons (Aschwanden 1996), although they are not trapped by magnetic mirrors.

3. The results that we have obtained for protons can give information on the electron dynamics, too. For $20 \mathrm{keV}$ electrons, the Larmor radius is much smaller than that of $1 \mathrm{MeV}$ protons, so that electron motion is adiabatic and magnetic mirroring in a trap of typical size $\lambda$ is obtained. On the other hand, it should be considered that the collisional mean free path of electrons is shorter than that of protons, so that 
collisions will influence electron trapping and electron motion along the loop.

4. In the case of either scatter-free or superdiffusive transport, or trapped motion, the proton dynamics is non-Gaussian, as evidenced by the computed values of the flatness, and the statistical description of transport requires the use of such ideas as Lévy random walk, long range correlations, fractal structures, and fractional Fokker-Planck equations (Bouchaud \& Georges 1990; Metzler \& Klafter 2000; Zaslavsky 2002; Zimbardo 2005).

5. We note that with fluctuation levels corresponding to $\delta B / B_{0}=0.05,0.1,0.2$, perpendicular transport is slow for most cases (i.e., except for $\ell / L=1$ ), and no (or very few) protons are able to exit the loop simulation box from the edges. This result implies that the loop is not feeding the corona with energetic protons, in agreement with the fact that for considered turbulence levels the majority of magnetic surfaces are closed (e.g., Zimbardo et al. 1995; Pommois et al. 1998; Zimbardo et al. 2000). In other words, larger values of $\delta B / B_{0}$ (as for instance $\delta B / B_{0}=0.4$ ) are needed (at least locally) to allow the particle leakage from the coronal loop.

We consider that the results reported here are relevant to the propagation of solar energetic particles, too, given that the transport of such particles in solar wind magnetic turbulence is sometimes considered to be scatter-free, and sometimes diffusive (e.g., Cane \& Erickson 2003; McKibben 2005; Qin et al. 2005; Zimbardo et al. 2006). Our results can help us to understand under what conditions each type of transport regime is to be expected. The strong dependence of the transport properties on $\rho / \lambda$ and on $\delta B / B_{0}$ emphasizes the importance of understanding what the actual features of magnetic turbulence in coronal loops are (e.g., Nigro et al. 2005; Galloway et al. 2006). In this study the electric fields created by the varying magnetic field or the local shear of magnetic field $(\nabla \times \mathbf{B})$ and the inclusion of the local resistivity have been ignored for simplicity. In real turbulent plasmas the transport and acceleration from localized electric fields coexist and the diffusion both in real and momentum space go hand in hand. Also, magnetic turbulence in the loop is likely to be anisotropic. These effects are left for a future study.

Acknowledgements. We thank G. Nigro for useful discussions, and the anonymous referee for useful suggestions. During her stay in Italy, M. Gkioulidou was partially supported by the European mobility program "Socrates". This research was partially supported by the European Union Research Training Network "Theory, Observation, and Simulation of Turbulence in Space Plasmas" (contract No. HPRN-cT-2001-00310), by the Italian Istituto Nazionale di Astrofisica (INAF), and by the Agenzia Spaziale Italiana (ASI). Numerical computations were performed at the High Performance Computing Center (HPCC) of the University of Calabria (Centro di Eccellenza MIUR).

\section{References}

Alexander, D. 1990, A\&A, 235, 431

Aschwanden, M. J. 1996, ApJ, 470, L69

Barat, C., Trottet, G., Vilmer, N., et al. 1994, ApJ, 425, L109

Bastian, T. S., Benz, A. O., \& Gary, D. E. 1998, ARA\&A, 36, 131

Buechner, J., \& Zelenyi, L. M. 1989, J. Geophys. Res., 94, 11821

Bouchaud, J.-P., \& Georges, A. 1990, Phys. Rep., 195, 127

Cane, H., \& Erickson, W. C. 2003, J. Geophys. Res., 108

Cargill, P. 2002, in SOLMAG: Magnetic coupling of the solar atmosphere, ESA SP-505, 245

Fleishman, G. D., \& Melnikov 1998, Phys. Uspek., 41, 1157

Fletcher, L. 1995, A\&A, 303, L9

Fletcher, L. 1996, A\&A, 310, 661

Fletcher, L. 1997, A\&A, 326, 1259

Galloway, L., Helander, P., \& MacKinnon, A. L. 2006, ApJ, 646, 615

Gkioulidou, M., Zimbardo, G., Pommois, P., Veltri, P., \& Vlahos, L. 2006, in Proceedings of SOHO17, 10 years of SOHO and beyond, ESA SP-617, P203

Klafter, J., Blumen, A., \& Shlesinger, M. F. 1987, Phys. Rev. A, 35, 3081

Klafter, J., Shlesinger, M. F., \& Zumofen, G. 1996, Phys. Today, 49, 33

Krall, N. A., \& Trivelpiece, A. W. 1972, Principles of Plasma Physics (New York: MacGraw-Hill), 302

Kundu, M. R., \& Vlahos, L. 1982, Space Sci. Rev., 32, 405

Mazur, J. E., Mason, G. M., Dwyer, J. R., et al. 2000, ApJ, 532, L79

McKibben, R. B. 2005, Adv. Space Res., 35, 518

Melrose, D. B., \& Brown, J. C. 1976, MNRAS, 176, 15

Metzler, R., \& Klafter, J. 2000, Phys. Rep., 339, 1

Metzler, R., \& Klafter, J. 2004, J. Phys. A: Math. Gen., 37, R161

Miller, J. A., Guessoum, N., \& Ramaty, R. 1990, ApJ, 361, 701

Miller, J. A., Cargill, P. J., Emslie, A. G., et al. 1997, J. Geophys. Res., 102/A7, 14631

Nigro, G., Malara, F., \& Veltri, P. 2005, ApJ, 629, L133

Petrosian, V. 1999, in Plasma turbulence and energetic particles in astrophysics, ed. M. Ostrowski, \& R. Schlickeisher, Observatorium Astromiczne, Uniwersytet jagiellonski, Krakow

Pommois, P., Zimbardo, G., \& Veltri, P. 1998, Phys. Plasmas, 5, 1288

Pommois, P., Veltri, P., \& Zimbardo, G. 1999, Phys. Rev. E, 59, 2244

Pommois, P., Veltri, P., \& Zimbardo, G. 2001, Phys. Rev. E, 63, 066405

Qin, G., Zhang, M., Dwyer, J. R., Rassoul, H. K., \& Mason, G. M. 2005, ApJ, 627,562

Reames, D. V., \& Tylka, A. J. 2002, ApJ, 575, L37

Ruffolo, D., Matthaeus, W. H., \& Chuychai, P. 2003, ApJ, 597, L169

Shlesinger, M., Zaslavsky, G., \& Frisch, U. 1995, Lévy Flights and Related Topics in Physics (Berlin: Springer)

Veronig, A. M., Brown, J. C., \& Bone, L. 2005, Adv. Space Res., 35, 1683

Vilmer, N., \& MacKinnon, A. L. 2003, in Energy conservation and particle acceleration in the solar corona, Proceedings of the CESPA Workshop, ed. K. L. Klein, Lecture Notes in Physics, 612

Zaslavsky, G. M. 2002, Phys. Rep., 371, 461

Zimbardo, G. 2005, Plasma Phys. Control. Fus., 47, B755

Zimbardo, G., Veltri, P., Basile, G., \& Principato, S. 1995, Phys. Plasmas, 2, 2653

Zimbardo, G., Veltri, P., \& Pommois, P. 2000, Phys. Rev. E, 61, 1940

Zimbardo, G., Pommois, P., \& Veltri, P. 2004, J. Geophys. Res., 109, A02113

Zimbardo, G., Pommois, P., \& Veltri, P. 2006, ApJ, 639, L91 